\title{
ON THE SENSITIVITY OF THE TUNNELING CURRENT TO ELECTRIC FIELD IN A MOSFET WITH TWO GATES
}

\author{
M. A. GRADO-CAFFARO* and M. GRADO-CAFFARO \\ C/Julio Palacios 11, 9-B, 28029-Madrid (Spain)
}

(Received 18 January 1999; In final form 9 March 1999)

\begin{abstract}
A theoretical model to evaluate the sensitivity of the tunneling current to the electric field in an $n$-channel MOSFET with two gates is proposed. This sensitivity is calculated in a real situation.
\end{abstract}

Keywords: Sensitivity; tunneling current; $n$-channel MOSFET

\section{INTRODUCTION}

It is well-known that MOSFETs with control gate and floating gate play an important role in EEPROM for low-voltage microcontrollers. This role arises mainly from the physical electronics involved in the above devices; in particular, the tunneling current through the oxide layer constitutes a relevant feature of these devices. This current is very sensitive to the electric field in the oxide layer so that evaluation of this sensitivity is very useful in order to estimate quantitatively the performance of the devices. The aim of this paper is to establish a parameter to estimate the above sensitivity; at this respect, we think that our model improves really the state of the art since to date not much has been done with respect to theoretical research on MOSFETs performance. At any rate, with respect to this research, we can mention Refs. [1-3].

${ }^{*}$ Corresponding author. 


\section{THEORETICAL MODEL}

First of all, we will consider the mathematical expression of the tunneling current density through the oxide layer of an $n$-channel MOSFET with two gates: the control gate and the floating gate. We will assume an $\mathrm{Al}-\mathrm{SiO}_{2}-\mathrm{Si}$ device so that the magnitude of the current density in question is given by the Fowler-Nordheim model, namely $[1-4]$ :

$$
J=\frac{e^{3} m_{o} E_{g}^{-3 / 2} E^{2}}{16 \pi^{2} \hbar m_{n}^{*}} \exp \left[-\frac{4\left(2 m_{n}^{*} E_{g}^{3}\right)^{1 / 2}}{3 e \hbar E}\right]
$$

where $e$ is the electron charge, $E$ denotes strength of the electric field in the oxide layer, $m_{o}$ stands for the electron rest-mass, $m_{n}^{*}$ is the tunneling electron effective mass, $\hbar$ is the reduced Planck's constant and $E_{g}$ is the barrier height of $\mathrm{Si}$ to $\mathrm{SiO}_{2}[1-4]$. Now we define the following quantity: $s \equiv J^{-1} d J / d E$ so that by formula (1) one obtains:

$$
s=E^{-1}\left(2+\frac{4\left(2 m_{n}^{*} E_{g}^{3}\right)^{1 / 2}}{3 e \hbar E}\right)
$$

By using the numerical values of the parameters involved in Eq. (2), including $m_{n}^{*} \approx 1.1 m_{o}$ and $E_{g}=4.35 \mathrm{eV}$ (room temperature) [3,5], it follows:

$$
s \approx E^{-1}\left(2+6.48 \times 10^{10} E^{-1}\right)
$$

where $E$ is expressed in $\mathrm{V} / \mathrm{m}$ and $s$ in $\mathrm{m} / \mathrm{V}$; we can conceive $s$ as a sensitivity parameter which measures the sensitivity of $J$ to $E$ (in Fig. 1, $s$ is depicted as a function of $E$ for a range of $E$-values of interest).

Notice that $s$ decreases as $E$ increases; a typical value of $E$ is $3 \times 10^{8} \mathrm{~V} / \mathrm{m}$ which corresponds to $V_{o x}=3 \mathrm{~V}$ and $t_{o x}=100 \AA$, where $V_{o x}$ stands for the voltage drop across the oxide layer and $t_{o x}$ is the oxide thickness (see previous references). The above numerical values correspond obviously to a uniform electric field $E \approx V_{o x} / t_{o x}$ which in practice constitutes a reasonable approximation. 


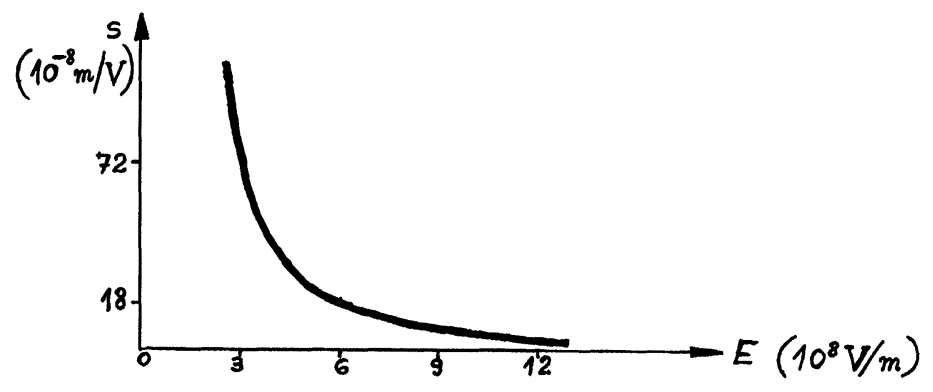

FIGURE 1 Plot of $s$ versus $E$ for a range of interest.

\section{CONCLUDING REMARKS}

The model described previously represents a useful approach to estimate the sensitivity of $J$ to $E$; our method may be regarded as a technique extrapolable to other situations in the context of high-speed electronics. In particular, by examining the $E$-dependence of $s$, it is very easy to see that for $E \ll 3 \times 10^{8} \mathrm{~V} / \mathrm{m} s$ varies sharply with $E$ although the situation corresponding to $E \ll 3 \times 10^{8} \mathrm{~V} / \mathrm{m}$ is irrelevant in practice; in contrast, $E$-values near $3 \times 10^{8} \mathrm{~V} / \mathrm{m}$ are relevant with a relatively remarkable variation of $s$ in terms of $E$; on the other hand, values between $3 \times 10^{8} \mathrm{~V} / \mathrm{m}$ and $12 \times 10^{8} \mathrm{~V} / \mathrm{m}$ present some interest.

\section{References}

[1] Grado-Caffaro, M. A. and Grado-Caffaro, M. (1998). Act. Pass. Electronic Comp., 20, $165-167$.

[2] Grado-Caffaro, M. A. and Grado-Caffaro, M. (1998). Act. Pass. Electronic Comp., 21, 57-60.

[3] Grado-Caffaro, M. A. and Grado-Caffaro, M. (1999). Eur. Phys. J. (Appl. Phys.), 5, $1-2$.

[4] Sze, S. M., Physics of semiconductor devices, second edition (Wiley, 1981).

[5] Grove, A. S., Physics and technology of semiconductor devices (John Wiley and Sons, 1967), p. 346. 

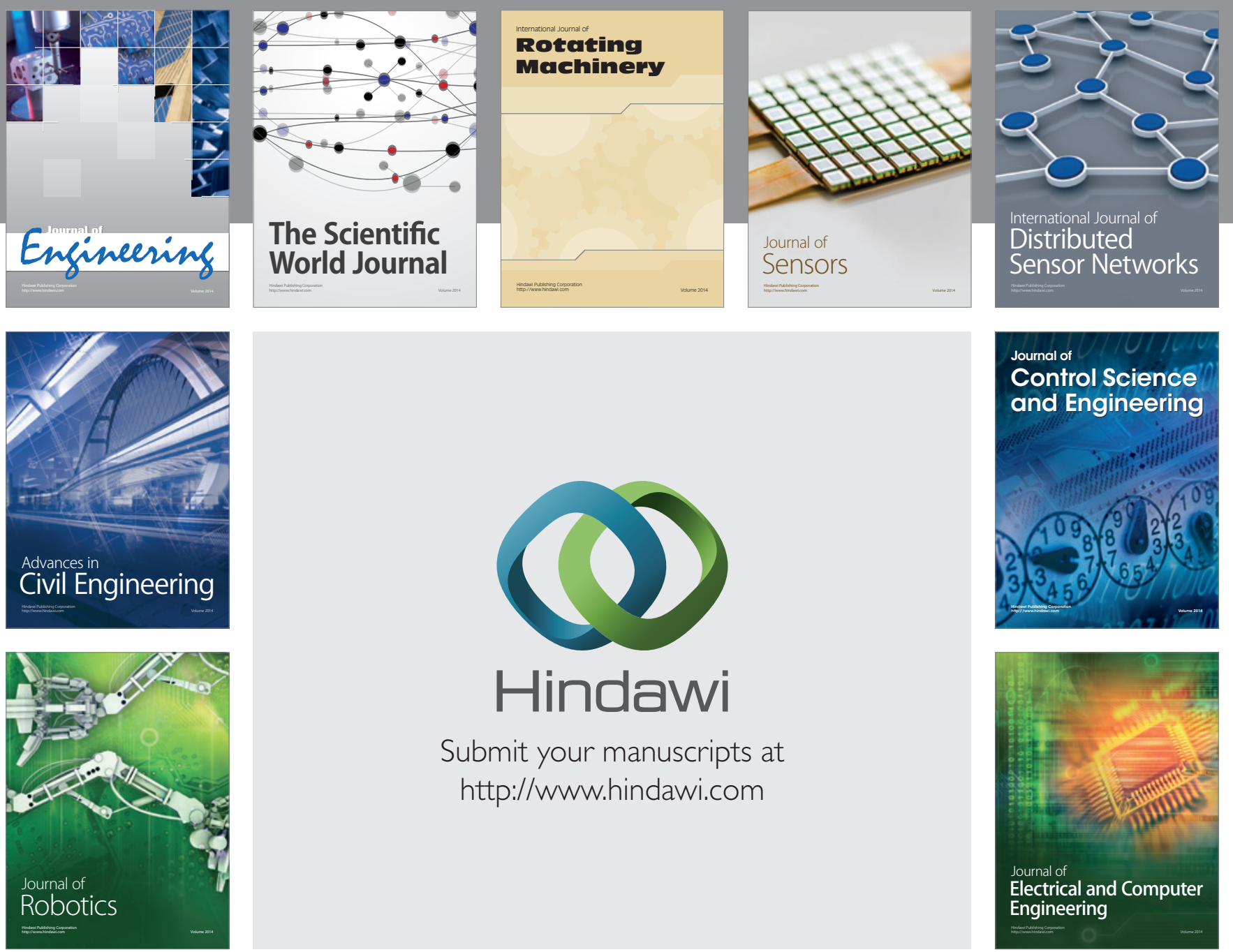

Submit your manuscripts at

http://www.hindawi.com
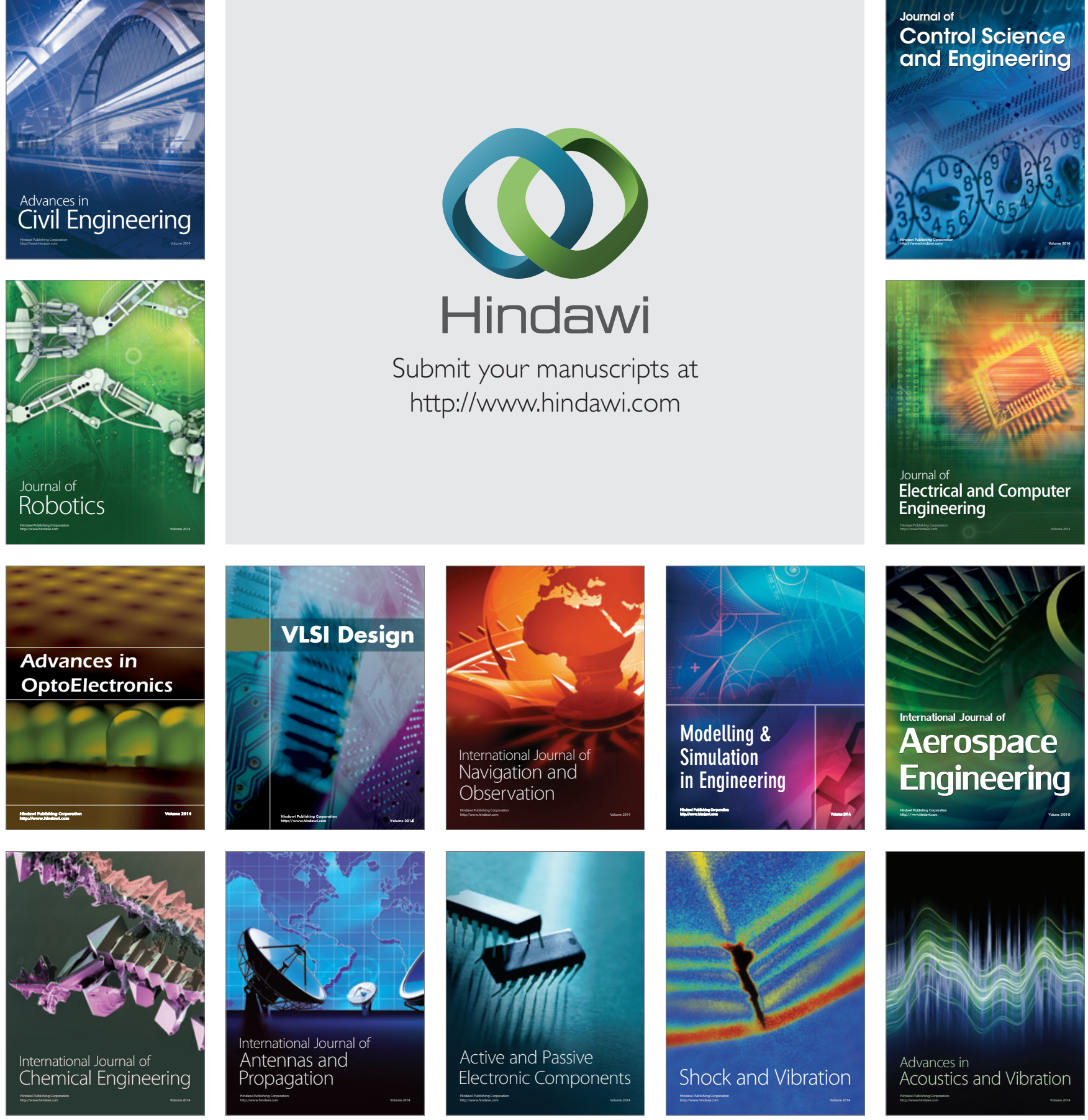